\title{
Visceral artery aneurysms: an atypical presentation of large-vessel vasculitis
}

\author{
Brendan Litt MD, Benedetto Mussari MD, Gabrielle Gauvin MD, David Alcoloumbre MD
}

Cite as: CMAJ 2017 March 27;189:E464-7. doi: 10.1503/cmaj.160023

A 79-year-old woman with atrial fibrillation, hypertension and hypothyroidism presented to the emergency department with syncope. She had previously experienced three minor syncopal episodes. A few months earlier, she had been prescribed rivaroxaban $15 \mathrm{mg} / \mathrm{d}$ after many years of warfarin therapy. On the day of presentation, she had woken feeling weak and fell to the floor, but managed to call 911. Following the initial assessment by emergency medical services she became unresponsive and briefly pulseless, and cardiopulmonary resuscitation was performed.

In the emergency department, the patient was resuscitated with several boluses of normal saline, one unit each of packed red blood cells, platelets and prothrombin complex concentrate. Her vital signs stabilized. Diffuse abdominal guarding and tenderness were noted on examination. No prior intra-abdominal surgery was reported, and there was no definite antecedent infection.

The patient's leukocyte count was 19.9 (normal 4.0-10.5) $\times$ $10^{9} / \mathrm{L}$, plasma lactate level 12.4 (normal $0.5-2.2$ ) $\mathrm{mmol} / \mathrm{L}$ and hemoglobin 83 (normal 120-160) g/L; her hemoglobin level had been $121 \mathrm{~g} / \mathrm{L}$ two months earlier. The international normalized ratio was 4.2 (normal 0.9-1.1). The platelet count and lipase level were within normal limits.

Contrast-enhanced computed tomography (CT) showed a large intraperitoneal hematoma centred on a $3-\mathrm{cm}$ pseudoaneurysm in the expected location of the pancreaticoduodenal arteries (Figure 1). There were several smaller fusiform aneurysms arising from the celiac and superior mesenteric branch vessels. In the descending thoracic and abdominal aorta, there was an intramural hematoma, with active filling of multiple penetrating ulcerations (Figure 2).

Interventional radiology was consulted for angiography and potential transcatheter embolization. Angiography confirmed the CT findings, including a $3-\mathrm{cm}$ pseudoaneurysm arising from the inferior pancreaticoduodenal artery. The artery was selected with a Progreat microcatheter (Terumo Interventional Systems), and the segments of the artery proximal and distal to the 3-cm ruptured pseudoaneurysm were embolized with five Nester embolization coils $3 \mathrm{~mm} \times 14 \mathrm{~cm}$ (Cook Medical) and two Interlock embolization coils $3 \mathrm{~mm} \times 12 \mathrm{~cm}$ (Boston Scientific) (Figure 3).

\section{KEY POINTS}

- Vasculitis should be considered as an uncommon cause of intra-abdominal hemorrhage; visceral artery aneurysms are a rare but potentially deadly consequence of vasculitis.

- Treatment of visceral artery aneurysms depends on the underlying cause: those due to vasculitis sometimes respond to corticosteroids, whereas corticosteroid treatment may worsen the outcome in mycotic or atherosclerotic aneurysms.

- Endovascular coiling and stent grafts have a growing role over more invasive surgical options in the management of visceral artery aneurysms.

Angiography of the celiac and superior mesenteric arteries showed many smaller aneurysms involving several branch vessels. Given that these aneurysms were not easily accessible and did not meet the size criteria of $2 \mathrm{~cm}$, they were not embolized. The interventional radiologist suspected vasculitis as the underlying cause. The smaller aneurysms were therefore expected to respond to medical therapy and did not require immediate intervention.

Following endovascular coiling, investigations showed an increase in nonspecific inflammatory markers, including a C-reactive protein level of 180.1 (normal $0-1.0$ ) $\mathrm{mg} / \mathrm{L}$ and an erythrocyte sedimentation rate of 60 (normal $0-27$ ) $\mathrm{mm} / \mathrm{h}$. Results of serologic evaluations were within normal limits, including tests for antineutrophil antibodies, anti-extractable nuclear antigen antibodies, perinuclear and cytoplasmic antineutrophil cytoplasmic antibodies, rheumatoid factor and cryoglobulin. Serologic tests for syphilis, hepatitis and Lyme disease were negative. Blood cultures were negative. Complement testing was not ordered.

With a presumptive diagnosis of vasculitis made by the consulting rheumatology service, the patient received methylprednisolone $250 \mathrm{mg} / \mathrm{d}$ intravenously for three days followed by a course of oral prednisone treatment. At follow-up one month later, she was asymptomatic. The temporal arteries were nontender on palpation. Follow-up CT showed stability of the mesenteric artery aneurysms and a decrease in size of the aortic intramural hematoma. At the three-month follow-up, the C-reactive protein level and erythrocyte sedimentation rate had fallen but remained elevated (69.5 $\mathrm{mg} / \mathrm{L}$ and $47 \mathrm{~mm} / \mathrm{h}$, respectively). 
Eight months following the initial episode of vasculitis, the patient had a sudden onset of central chest pain radiating to both scapulae. Her systolic blood pressure was $220 \mathrm{~mm} \mathrm{Hg}$ at triage in the emergency department. Computed tomography showed a new intramural hematoma and aortic ulceration in the descending thoracic aorta. Tests for inflammatory markers were not ordered. Given the substantial morbidity and mortality associated with open aortic repair, a decision was made to proceed with thoracic endovascular aortic repair. The following day, a two-component Zenith Alpha endograft (Cook Medical) was successfully placed in the descending thoracic aorta through a femoral approach. Coverage of the stent was from the left subclavian artery to just above the celiac origin.
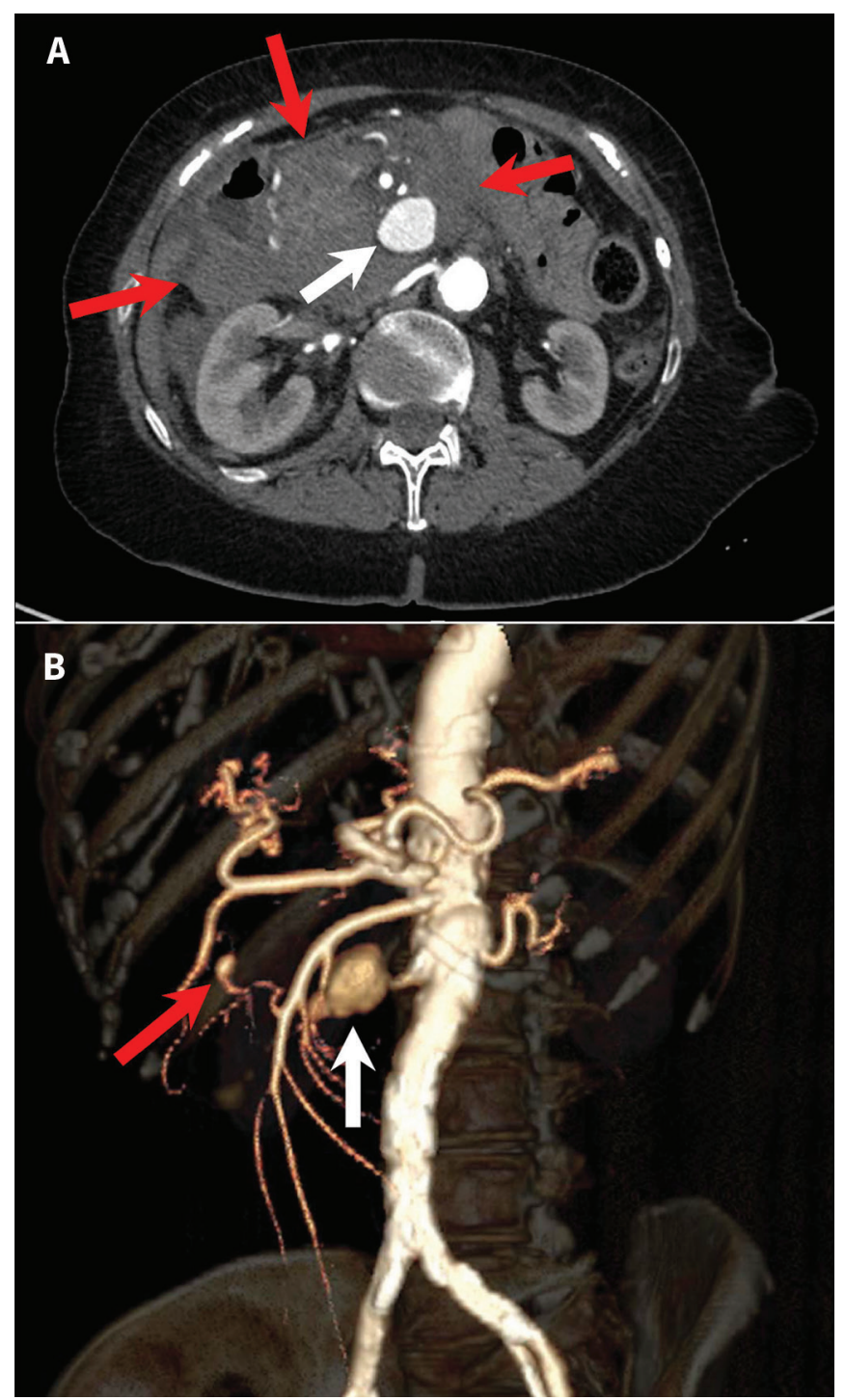

Figure 1: (A) Axial abdominal computed tomography (CT) angiogram, showing a large intraperitoneal hematoma (red arrows) in a 79-year-old woman who presented with syncope. Within the hematoma is a 3-cm pseudoaneurysm (white arrow) posterior to the superior mesenteric artery. There are several additional smaller visceral artery aneurysms (not visualized). (B) Three-dimensional volume rendering of the abdominal CT data, showing the anatomy of the largest aneurysm (white arrow) arising from the inferior pancreaticoduodenal branch of the superior mesenteric artery. Another small aneurysm is seen arising from a different branch of the superior mesenteric artery (red arrow).
The patient's postoperative course was unremarkable. Followup CT scans seven months after the thoracic endovascular aortic repair showed improvement in the aortic intramural hematoma and resolution of the mesenteric artery aneurysms. Follow-up CT scans 16 months after the procedure were unchanged.

\section{Discussion}

Nontraumatic hemoperitoneum is rare and often catastrophic and requires a thorough but swift radiologic search for the underlying cause. It is most often an unexpected finding in the workup of patients with acute abdomen. A basic approach includes assessment for gynecologic causes, hepatic neoplasm, splenic rupture and aneurysm rupture, listed in decreasing order of frequency. ${ }^{1}$

Visceral artery aneurysm is an uncommon cause of nontraumatic intra-abdominal hemorrhage. ${ }^{1}$ The rarity of this entity has the unfortunate consequence of late diagnosis, often at the time of rupture or other complications. In one case series, mortality was reported to be $25 \%$. $^{2}$

In patients presenting with intra-abdominal hemorrhage of unproven cause on ultrasound, unenhanced $\mathrm{CT}$ or portal venous phase CT, an arterial phase CT scan must be performed when clinically feasible to evaluate the source of bleeding. This is often the only contrast-enhanced phase that will reveal visceral artery aneurysm. Successful identification has implications for both immediate management and long-term prognosis.

Although many causes have been reported for visceral artery aneurysm, atherosclerosis is the most common. Prior surgery, pancreatitis, acute and chronic infections, fibromuscular dysplasia, connective tissue disorders, vasculitis, neurofibromatosis, radiation therapy and segmental arterial mediolysis represent alternative causes of increasing rarity. ${ }^{3,4}$

Visceral artery aneurysm is rarely associated with concurrent aortic disease, with very few cases reported in the literature. Our patient's anticoagulation with rivaroxaban may have played a role. Rupture of the pancreaticoduodenal aneurysm with aortic dissection has been reported in the setting of anticoagulation. ${ }^{5}$

The clinical picture of our patient favours atypical mediumand large-vessel vasculitis of unknown subtype. Nonspecific inflammatory markers remained elevated three months after presentation, which supports a diagnosis of vasculitis. In addition, the corticosteroid treatment appeared to ameliorate the course of the disease, with resolution of the visceral artery aneurysms at interval follow-up imaging. There were no specific clinical findings (e.g., vision change, abnormal temporal artery on palpation, new headache) to suggest giant-cell arteritis, ${ }^{6}$ a common vasculitis in this age group. Histology would provide a more definitive diagnosis, but it is rarely available antemortem.

Segmental arterial mediolysis, a poorly understood noninflammatory mimic of vasculitis, was an additional consideration in this case. It is an exceedingly rare (and possibly underdiagnosed) entity. Since its first description in 1976, about 50 cases were reported as of $2010 .{ }^{3}$ Cases of segmental arterial mediolysis with elevated erythrocyte sedimentation rate and C-reactive protein level have occasionally been reported, ${ }^{7}$ as has aortic involvement. ${ }^{8}$ 
We did not entertain additional causes of visceral artery aneurysm. Negative findings on infection workup, including negative results of blood cultures and serologic testing for syphilis and Lyme disease, precluded a diagnosis of mycotic aneurysms. The patient's age, distribution of arterial involvement on imaging, clinical findings and clinical course were likewise not compatible with Ehlers-Danlos syndrome or fibromuscular dysplasia. The negative serologic mark-

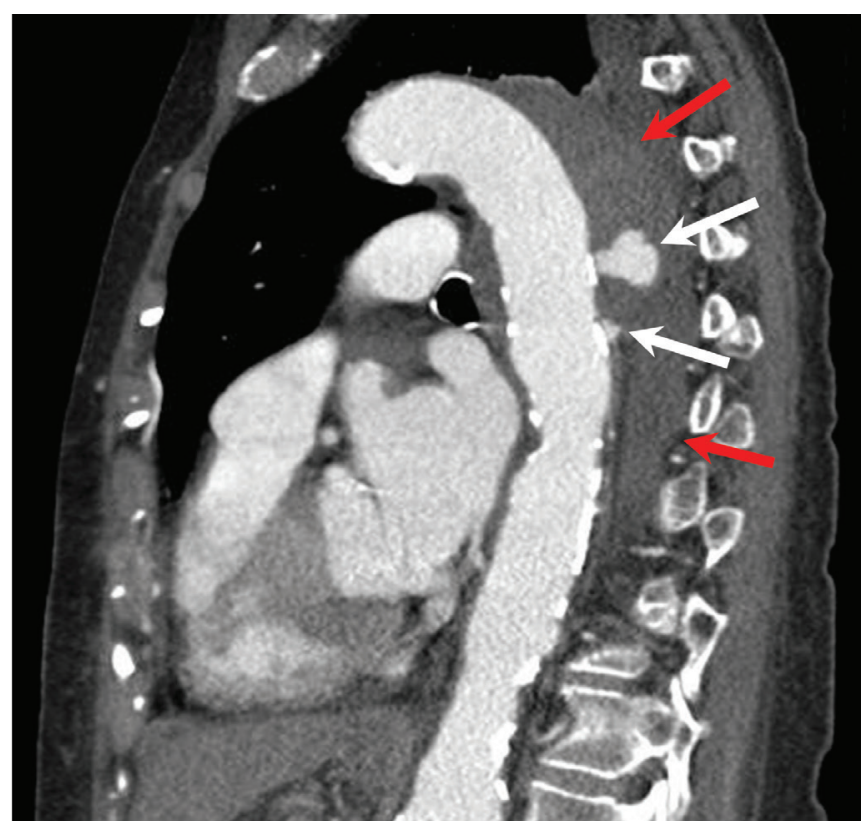

Figure 2: Sagittal computed tomography angiogram of the patient's chest. In the descending thoracic aorta, a posteriorly located intramural hematoma (red arrows) is visible, with contrast medium filling multiple penetrating ulcerations (white arrows). The ulcerations and intramural hematoma extended below the diaphragmatic hiatus (not pictured). ers for antineutrophil cytoplasmic antibodies and hepatitis B work rule out vasculitis associated with antineutrophil cytoplasmic antibodies and polyarteritis nodosa, respectively. Finally, mycotic aneurysms and those resulting from connective tissue disorders would not be expected to resolve spontaneously, particularly following a course of corticosteroid treatment.

\section{Treatment}

The management of visceral artery aneurysms remains controversial. Prior reports suggest treatment of asymptomatic aneurysms that are greater than $2 \mathrm{~cm}$ in diameter or that show rapid growth, ${ }^{9}$ whereas others suggest treatment of visceral artery aneurysms of any size. ${ }^{2}$ Aneurysms of the pancreaticoduodenal arcade are especially prone to rupture, regardless of their size. ${ }^{10}$ Clinical trials are often small (fewer than 30 patients), owing to the relative rarity of these lesions, and follow-up is limited. ${ }^{2}$

Traditionally, visceral artery aneurysms have been treated by open surgical repair. The role of endovascular coiling has expanded over the years, particularly in unstable patients and those with substantial medical comorbidities, ${ }^{2,3}$ and the procedure is currently considered the treatment of choice. ${ }^{3}$

Inflammatory aneurysms present a unique set of challenges. Although corticosteroid therapy is associated with an increased rate of rupture in noninflammatory aneurysms, ${ }^{3}$ it is the mainstay of treatment of inflammatory vasculitides. ${ }^{4}$

The patient's inflammatory thoracic aortic disease was treated with thoracic endovascular aortic repair, a less invasive alternative to open aortic repair. The use of endovascular repair in inflammatory thoracic aortic disease is not well studied, owing to the rarity of the disease. Its role in abdominal aortic disease is better established and provides a framework for treating thoracic aortic disease. Endovascular repair of inflammatory abdominal

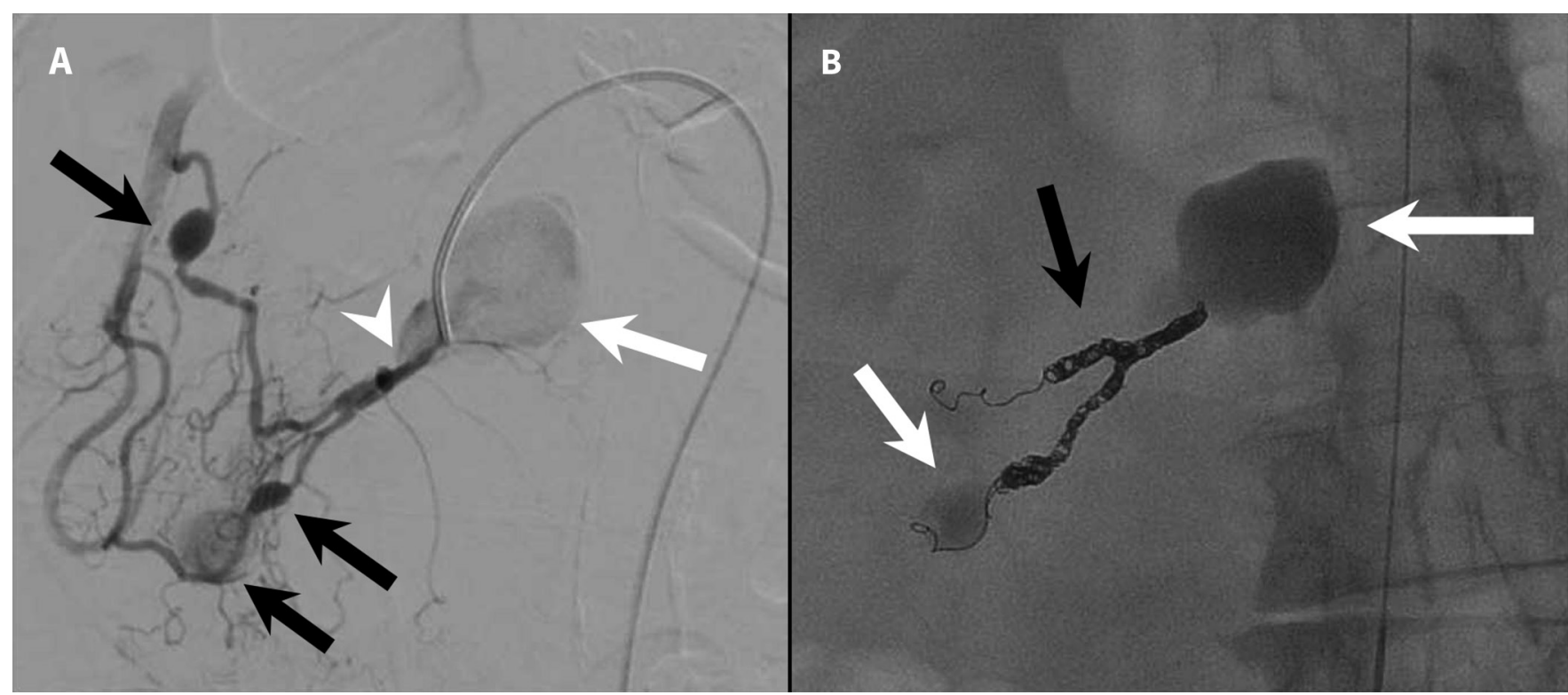

Figure 3: (A) Digital subtraction angiogram: the inferior pancreaticoduodenal artery has been subselected from the superior mesenteric artery. The image shows a large pseudoaneurysm (white arrow) arising from the proximal inferior pancreaticoduodenal artery, which corresponds to the aneurysm found on computed tomography. The neck of the pseudoaneurysm (arrowhead) is not clearly visible. Smaller fusiform aneurysms are noted in distal branches (black arrows). (B) Completion angiogram following endovascular coiling (black arrow) of the inferior pancreaticoduodenal artery supplying the largest pseudoaneurysm. The aneurysms are filled with stagnant contrast medium, which indicates successful embolization. 
aortic aneurysms was previously cautioned against because of concerns of persistent retroperitoneal inflammation. However, a recent case-control study involving 67 patients supported the use of endovascular aortic repair in this setting, ${ }^{11}$ and alternative treatment options are scarce in patients with acute aortic disease.

\section{References}

1. Kasotakis G. Spontaneous hemoperitoneum. Surg Clin North Am 2014;94:65-9.

2. Carr SC, Mahvi DM, Hoch JR, et al. Visceral artery aneurysm rupture. J Vasc Surg 2001;33:806-11.

3. Pitton MB, Dappa E, Jungmann F, et al. Visceral artery aneurysms: incidence, management, and outcome analysis in a tertiary care center over one decade. Eur Radiol 2015;25:2004-14.

4. Baker-LePain JC, Stone DH, Mattis AN, et al. Clinical diagnosis of segmental arterial mediolysis: differentiation from vasculitis and other mimics. Arthritis Care Res (Hoboken) 2010;62:1655-60.

5. Taniai S, Nagai W, Shimizu H, et al. Rupture of pseudoaneurysm of the pancreaticoduodenal arcade after acute aortic dissection in a patient on anticoagulant therapy. J Cardiol Cases 2011;3:e103-e105.

6. Hunder GG, Bloch DA, Michel BA, et al. The American College of Rheumatology 1990 criteria for the classification of giant cell arteritis. Arthritis Rheum 1990; 33:1122-8.

7. Kalva SP, Somarouthu B, Jaff MR, et al. Segmental arterial mediolysis: clinical and imaging features at presentation and during follow-up. J Vasc Interv Radiol 2011;22:1380-7.

8. Ushijima T, Izumo A, Matsumoto T, et al. Pancreaticoduodenal artery pseudoaneurysm caused by segmental arterial mediolysis: a case report of surgical treatment. Ann Vasc Dis 2013;6:198-201.

\section{Competing interests: None declared.}

This article has been peer reviewed.

The authors have obtained patient consent.

Affiliations: Departments of Diagnostic Radiology (Litt, Mussari) and Surgery (Gauvin, Alcoloumbre), Kingston General Hospital, Queen's University, Kingston, Ont.
9. Hirsch AT, Haskal ZJ, Hertzer NR, et al. ACC/AHA 2005 practice guidelines for the management of patients with peripheral arterial disease (lower extremity, renal, mesenteric, and abdominal aortic): a collaborative report from the American Association for Vascular Surgery/Society for Vascular Surgery, Society for Cardiovascular Angiography and Interventions, Society for Vascular Medicine and Biology, Society of Interventional Radiology, and the ACC/AHA Task Force on Practice Guidelines (Writing Committee to Develop Guidelines for the Management of Patients With Peripheral Arterial Disease): endorsed by the American Association of Cardiovascular and Pulmonary Rehabilitation; National Heart, Lung, and Blood Institute; Society for Vascular Nursing; TransAtlantic Inter-Society Consensus; and Vascular Disease Foundation. Circulation 2006;113:e463-654.

10. de Perrot M, Berney T, Deléaval J, et al. Management of true aneurysms of the pancreaticoduodenal arteries. Ann Surg 1999;229:416-20.

11. Stone WM, Fankhauser GT. Inflammatory aneurysms treated with EVAR. Semin Vasc Surg 2012;25:227-31.

The section Cases presents brief case reports that convey clear, practical lessons. Preference is given to common presentations of important rare conditions, and important unusual presentations of common problems. Articles start with a case presentation (500 words maximum), and a discussion of the underlying condition follows (1000 words maximum). Visual elements (e.g., tables of the differential diagnosis, clinical features or diagnostic approach) are encouraged. Consent from patients for publication of their story is a necessity. See information for authors at www.cmaj.ca.
Contributors: Benedetto Mussari contributed to the design and conception of the report. Brendan Litt, Gabrielle Gauvin and David Alcoloumbre drafted the manuscript and contributed to the critical review of the literature. Brendan Litt and Benedetto Mussari revised the manuscript. All of the authors approved the final version to be published and agreed to act as guarantors of the work.

Correspondence to: Brendan Litt, 13bel2@queensu.ca 